Provided by the author(s) and University of Galway in accordance with publisher policies. Please cite the published version when available.

\begin{tabular}{|c|l|}
\hline Title & $\begin{array}{l}\text { Nested circulation modelling of inter-tidal zones: details of a } \\
\text { nesting approach incorporating moving boundaries }\end{array}$ \\
\hline Author(s) & Nash, Stephen; Hartnett, Michael \\
\hline $\begin{array}{c}\text { Publication } \\
\text { Date }\end{array}$ & 2010 \\
\hline $\begin{array}{c}\text { Publication } \\
\text { Information }\end{array}$ & $\begin{array}{l}\text { Nash, S,Hartnett, M (2010) 'Nested circulation modelling of } \\
\text { moving boundaries'. Ocean Dynamics, 60 :1479-1495. }\end{array}$ \\
\hline $\begin{array}{c}\text { Link to } \\
\text { publisher's } \\
\text { version }\end{array}$ & http://dx.doi.org/10.1007/s10236-010-0345-8 \\
\hline $\begin{array}{c}\text { Item record } \\
\text { herting://hdl.handle.net/10379/3475 }\end{array}$ \\
\hline
\end{tabular}

Some rights reserved. For more information, please see the item record link above. 


\title{
Nested Circulation Modelling of Inter-tidal Zones: Details of a Nesting Approach Incorporating Moving Boundaries
}

\author{
S. Nash and M. Hartnett
}

Civil Engineering, College of Engineering and Informatics, National University of Ireland, Galway, Ireland.

\author{
Corresponding Author: Dr Stephen Nash \\ Email: stephen.nash@nuigalway.ie \\ Phone: +35391493738 \\ Fax: $\quad+35391497507$
}

\begin{abstract}
Nested circulation models developed to date either exclude the flooding and drying process or prohibit flooding and drying of nested boundaries; they are therefore ill-suited to the accurate modelling of intertidal areas. The Authors have developed a nested model with moving boundaries which permits flooding and drying of both the interior domain and the nested boundaries. The model uses a novel approach to boundary formulation; ghost cells are incorporated adjacent to the nested boundary cells so that the nested boundaries operate as internal boundaries. When combined with a tailored adaptive interpolation technique, the approach facilitates a dynamic internal boundary. Details of model development are presented with particular emphasis on the treatment of the nested boundary. Results are presented for Cork Harbour, a natural coastal system with an extensive inter-tidal zone and a complex flow regime which provided a rigorous test of model performance. The nested model was found to achieve the accuracy of a high resolution single grid model for a much lower computational cost.
\end{abstract}

\section{Keywords:}

Nesting; circulation model; ghost cells; internal boundary; flooding and drying; moving boundary 


\section{Introduction}

A common problem in coastal modelling is the correct location of open boundaries; they must be located such that their conditions will not adversely affect model predictions within the area of interest (AOI). This often leads to a situation which requires a large computational domain, of which the AOI comprises only a small percentage. For a structured grid, a high spatial resolution requirement in the AOI can lead to excessively high computational costs. This may necessitate the use of a lower resolution resulting in a loss in accuracy. A common solution to this problem is the use of a nesting method which allows the increase of spatial resolution in a sub-region of the model domain without incurring the computational expense of fine resolution over the entire domain.

In coastal models, the problem of insufficient resolution and poor accuracy is typically associated with areas of complex bathymetry and irregular coastlines; these areas are generally in the inter-tidal zone. A nested domain in such an area will require simulation of flooding and drying both within the domain and along its boundaries. Most nested models developed to date do not incorporate flooding and drying as they have been specifically developed for ocean basin or large-scale regional applications where flooding and drying is not important. Those nested models that do incorporate flooding and drying, e.g. Mike21 (DHI Software, 2007), prohibit the flooding and drying of open boundaries as it tends to cause instabilities in the model solution. This is primarily because flooding and drying of an open boundary necessitates a moving boundary where the boundary extents shift during the course of a simulation so as to exclude grid cells upon drying and reinstate those same grid cells upon flooding.

The nested model developed by the Authors was designed to facilitate moving boundaries for the nested domains; to the Authors' knowledge it is the first nested circulation model to do so. The model is therefore particularly applicable to the high resolution modelling of the inter-tidal zone. Ghost cells are specified adjacent to nested boundaries so that the governing equations at the nested boundary grid cells are formulated in a similar manner to interior grid cells; the nested boundaries thus become internal boundaries. The internalisation of the boundaries, when combined with an adaptive interpolation technique, enables the stable flooding and drying of boundary grid cells. The internal boundary formulations also give improved simulation of the effect of the external environment on the internal solution resulting in improved conservation of mass and momentum between the coarse and fine grids and thus improved model performance.

The DIVAST (Depth Integrated Velocity and Solute Transport) model was chosen for nested development as it contains a robust flooding and drying routine (for details see Falconer and Chen (1991)). DIVAST is a well-accepted, two-dimensional, depth-integrated model which allows simulation of hydrodynamics, solute transport and water quality. The hydrodynamic module is based on the solution of the depth integrated Navier-Stokes equations and includes the effects of local and advective accelerations, the rotation of the earth, barotropic and free surface pressure gradients, wind action, bed resistance and a simple mixing length turbulence model. Solute transport and water quality are computed 
using the general depth integrated advection-diffusion equations and incorporate local and advective effects, turbulent dispersion and diffusion, wind effects, source and sink inputs and decay and kinetic transformation processes.

The model was developed using idealised rectangular harbour bathymetries. Post-development it was important to assess model performance in a natural environment. Cork Harbour, located on the southwest coast of Ireland, was chosen as the test case. Its long, irregular coastline and extensive intertidal zone create a complex circulation regime; it therefore provided a rigorous test of model performance.

An overview of the nested model is presented outlining the main features of the nesting technique adopted. Particular emphasis is placed on the treatment of the nested boundary which was designed to reduce error generation within the nested domain. Aspects of the spatial interpolation scheme, the boundary condition and the use of ghost cells in the boundary formulations are presented. Finally, results are presented for Cork Harbour demonstrating both the accuracy of the new model for a natural system and its computational efficiency.

\section{Overview of Nesting Technique}

Nested models are categorised as either one-way (passive) models or two-way (interactive) models. Oneway models use output from a coarse grid model to provide boundary conditions for the nested fine grid model. The coarse grid therefore affects the fine grid but there is no mechanism by which the evolution in the fine grid can affect the coarse grid (and hence its own boundary conditions). Two-way models, in addition to providing boundary conditions for the fine grid, allow the evolution within the fine grid to influence the evolution on the coarse grid. In oceanography, one-way nesting was first implemented by Spall and Robinson (1989) and has since become an established method for studying highly resolved subdomains. In later years, Spall and Holland (1991) developed the first two-way nested oceanographic model and numerous two-way nested models have since been developed (e.g. Fox and Maskell, 1995; Ginis et al., 1998; Barth et al., 2005). Although there are advantages to the interactive system they are necessarily more complicated and computationally expensive (Spall and Holland, 1991).

While much recent research has focussed on two-way nesting, one-way nesting of oceanographic models is still very much an active research area. One-way nesting techniques are best-suited to studying localised phenomena such as land-sea interactions in the coastal zone. In particular, one-way nesting techniques are favoured for limited-area operational modelling where downscaling to coastal areas is required (e.g. Korres and Lascaratos, 2003; Leitao et al., 2005; Nittis et al., 2006; Staneva et al., 2009; Holt et al., 2009). In such cases, feedback from the limited-area models to the larger-scale ocean models is not required and two-way nesting techniques are therefore unnecessary. The present research concerned down-scaling to an AOI in the coastal zone. The interaction of the high resolution AOI with the low resolution parent domain was not of interest; a one-way nested approach was therefore most suitable. 
The nested model allows finer spatial resolution to be focused over an AOI by introducing an additional grid (or grids) into the simulation. The present model consists of one outer coarse grid (the parent grid, PG) into which one or more inner fine grids are nested (the child grids, CG). Studies have shown that acceptable results are obtained for spatial nesting ratios $\left(\Delta x_{\text {parent }} / \Delta x_{\text {child }}\right)$ of $3: 1$ and 5:1 (e.g. Spall and Holland (1991)) but that substantial degradation in model performance occurs for higher nesting ratios (Barth et al., 2005).The model has been developed to enable multiple nesting so that each child may also be a parent to further children. In this way numerous levels of nesting can be specified and child grids may be telescoped to achieve virtually any required spatial resolution.

The interaction between a parent and child model is crucial to the accuracy of the nested solution. In general, the interaction involves the following processes: The parent model is first advanced to the next time level. Water surface elevations and velocities are then extracted along the nested boundaries of the child grid. Since the grid resolutions differ, the parent and child grid solutions at the boundary must be blended. The boundary operator is responsible for blending the solutions and is a critical element of a nested model. The boundary data extracted from the parent grid solution is interpolated in space (and usually in time) to obtain a full set of boundary data for the child. These data are then assigned to the child grid according to some boundary condition and the child model is advanced to the time level of the parent. A boundary operator thus comprises an interpolation scheme and a boundary condition.

The nesting procedure used by the model is presented in graphical form in Figure 1. For clarity the procedure is only presented for a single level of nesting and boundary data is only shown for water surface elevations, $\zeta$. The procedure may be summarised as follows:

1. advance parent model one full timestep to time level $\left(t+\Delta t_{p}\right)$

2. extract required boundary data from parent grid and interpolate (in time and space) to required time levels for child grid $\left(t+1 / 2 \Delta t_{c}\right)$ and $\left(t+\Delta t_{c}\right)$

3. integrate child grid one full timestep to time level $\left(t+\Delta t_{c}\right)$

4. repeat Steps 2 and 3 until time level of child grid matches that of parent grid $\left(t+\Delta t_{p}\right)$

5. return to Step 1 and continue

The order of time integration within the model is also shown in Figure 1. The parent grid may only be advanced in time when the child has been advanced to the time-level of its parent. The model uses the ADI solution technique to solve the governing continuity and momentum equations; this requires each timestep be split into two. This does not affect the order of time integration as the parent grid is advanced by one full timestep before any computations begin in the child. However, the time-splitting does affect the temporal interpolation process as the child grid requires boundary data at each half-timestep as shown. 


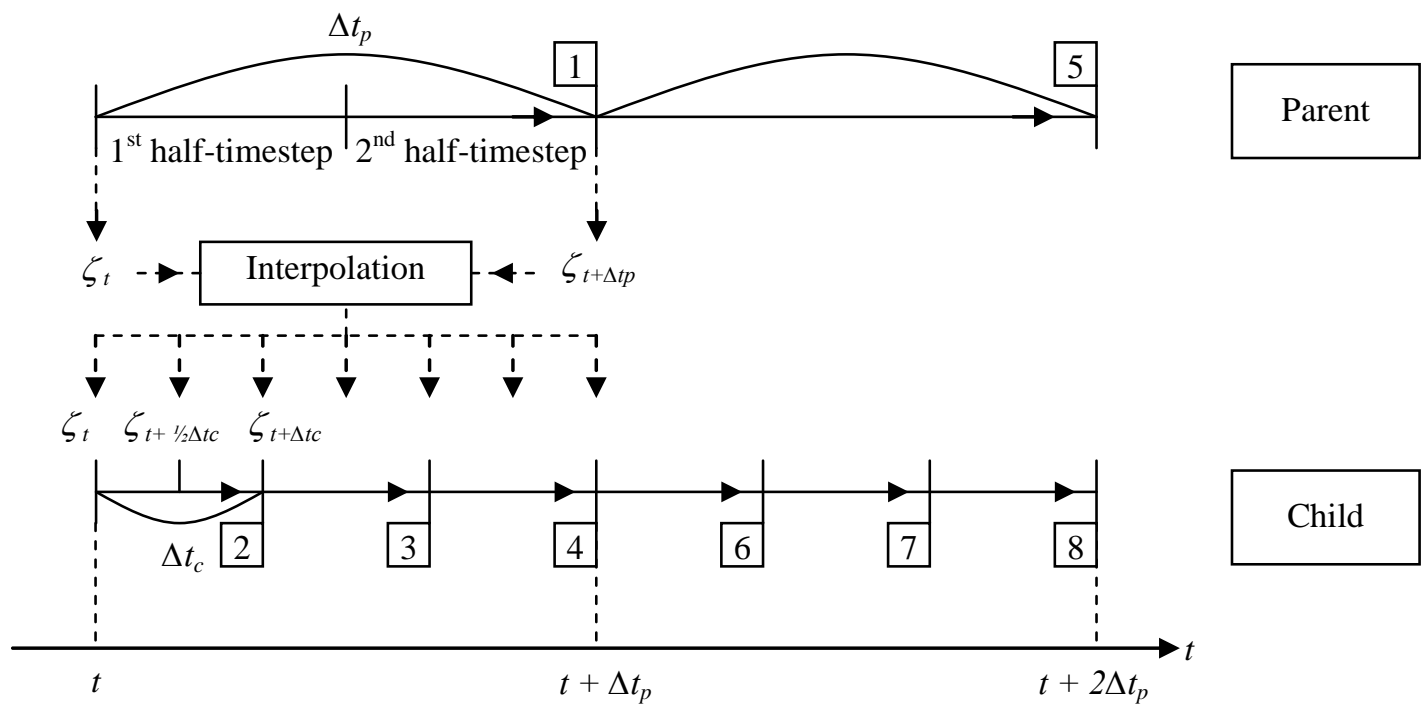

Fig 1 Schematic of the nesting procedure ( $p$ and $c$ signify parent and child grids)

\section{Treatment of the Nested Boundary}

Errors are introduced into a one-way nested model at the interface between the parent and child grids. It is widely-accepted that there are two sources of error at the boundary interface: (1) boundary specification errors and (2) boundary formulation errors (Koch and McQueen, 1987). The former are errors arising from the use of incorrect boundary data; they are therefore the result of inaccuracies in the PG solution at the CG boundary. The latter are errors arising from problems in blending the solutions of the parent and child grids at the boundary; they are therefore a result of the boundary operator. However, the Authors suggest that a third source of errors exists, arising from the mathematical formulations of the governing equations on the nested boundary. Simplifications are sometimes made to the formulations of the governing equations at a nested boundary, as opposed to at interior grid points, due to finite differencing requirements. As the CG model assimilates the boundary data through these mathematical formulations, their simplification can result in a source of error distinct from the boundary operator. The Authors therefore propose a more correct description of error sources as follows:

(1) boundary specification (BS) errors: result from incorrect boundary data

(2) boundary operator (BO) errors: result from a poorly designed boundary operator

(3) boundary formulation (BF) errors: result from simplified boundary formulations

BS errors may be reduced by locating nested boundaries in areas of high PG accuracy. However, this is not always easy in coastal models as errors tend to be highest in areas of land-sea interaction. In addition, the location of nested boundaries in inter-tidal zones requires a moving boundary where sections of the boundary can alternately flood and dry. The main focus of model development was the design of a moving nested boundary which aimed to minimise the three error sources defined. 


\subsection{Boundary Operator}

Zhang et al. (1986) suggest that an optimal boundary operator has the following properties:

(1) all resolvable waves must propagate across the boundary interface smoothly without generating significant noise

(2) properties such as mass and momentum, exchanged between the two grids, must be conserved The boundary operator was designed and tested with a view to satisfying these two criteria, thereby reducing $\mathrm{BO}$ errors.

\section{The Interpolation Scheme}

The different resolutions of the parent and child grids mean that data from the parent grid must be interpolated in both space and time in order to obtain a full set of boundary data for the child. An unsuitable, or inaccurate, interpolation scheme can lead to errors as a result of loss of mass or momentum, or imperfect reproduction of the parent waveform. Linear interpolation is almost always employed for temporal interpolation (e.g. Miyakoda and Rosati (1977); Pullen and Allen (2001); Korres and Lascaratos (2003)) and was therefore used in the model. Linear and quadratic schemes are most often used for spatial interpolation; however, a number of schemes were evaluated in relation to the conservation of mass and momentum. Four schemes were tested: 1) a zeroth order scheme (ZS), 2) a linear scheme (LS), 3) a massconserving quadratic scheme (QS), and 4) an inverse distance weighted scheme (IS). Details of the interpolation formulae are provided in Appendix A. Results from the testing of the interpolation scheme are presented in Section 4.1. The linear interpolation scheme was found to be most accurate; it was therefore implemented in the model.

\section{The Boundary Condition}

The choice of relevant boundary conditions for nested models is a difficult one and has been the focus of many studies. Blayo and Debreu (2005) and Marcehsiello et al. (2001) suggest that the best approach is one where incoming and outgoing fluxes are treated separately. The boundary should in effect specify incoming data and evacuate outgoing data. While the application of such a boundary condition works well in idealised experiments it is more difficult to implement in the complex flows of natural systems where, for example, boundaries may exhibit recirculation.

The most common implementations of boundary conditions in nested models are either relaxation or radiation conditions. Three different types of boundary conditions were tested during model development:

- a Dirichlet (or clamped) condition

- a flow relaxation condition

- a radiation condition

The Dirichlet condition is the most basic form of the relaxation condition. The parent model solution $\phi_{p}$ is directly imposed on the child grid solution $\phi_{c}$ along the nested boundary $\Gamma$ such that: 


$$
\phi_{c}=\phi_{p}
$$

While this condition usually ensures conservation of model properties, it can also cause reflection of outgoing waveforms if $\phi_{p}$ is incompatible with the outgoing solution $\phi_{c}$. Incompatibilities will occur when $\phi_{p}$ is inaccurate.

The flow relaxation condition attempts to reduce the wave reflection generated by the Dirichlet condition. Here the CG domain is extended by defining an additional domain adjacent to the CG boundary, known as the relaxation zone or sponge layer. The CG solution is relaxed towards the PG solution in the sponge layer so that incompatibilities between the solutions are minimised. The solution within the sponge layer is calculated according to:

$$
\phi_{s}=(1-\tau) \phi_{c}+\tau \phi_{p}
$$

where $\tau$ is a relaxation function which decreases from 0 on $\Gamma$ (the inner boundary of the sponge layer), to 1 on the outer boundary of the sponge layer.

Finally, radiation conditions attempt to solve the problem of outgoing wave reflection by allowing them to radiate freely out through the boundary. Radiation conditions are derived from the Sommerfeld radiation condition and assume that the outgoing solution propagates through the boundary in a wave-like form according to:

$$
\frac{\partial \phi_{c}}{\partial t}+C_{\phi} \frac{\partial \phi_{c}}{\partial n}=0
$$

where $C_{\phi}$ is the phase speed of the outgoing wave and $n$ is the direction normal to the boundary $\Gamma$. The radiation condition is only applied to outgoing variables. Incoming boundary data is therefore interpolated from $\phi_{p}$ while outgoing boundary data is calculated using (3). The determination of $C_{\phi}$ can prove problematic in complex flows (Blayo and Debreu, 2005) where $C_{\phi}$ is not constant along $\Gamma$. This was found to be the case during model development. A radiation condition based on advective extrapolation was thus employed where boundary values for outgoing variables were extrapolated from the child grid solution. Due to space limitation, results from the boundary condition testing are only summarised herein. For the complete details of the implementation and testing of the boundary conditions the reader is referred to Nash (2010).

As expected, the radiation condition was found to minimise outgoing wave reflection when incompatibilities between the child and parent solutions were large. However, neither mass nor momentum was accurately conserved for incoming waveforms, particularly in locations where the flow 
was strongly influenced by momentum. In addition, instabilities were also observed adjacent to the boundary at times of complex flows, i.e. when waveforms were simultaneously flowing inward and outward across the boundary. Blayo and Debreu (2005) have questioned the relevance of radiation boundaries in such complex flows and such boundary conditions have proved to give rather poor results in several comparative studies (e.g. Roed and Cooper (1987); Palma and Matano (1998); Nycander and Doos (2003)). Where incompatibilities between the child and parent solution were small, the Dirichlet condition was found to give improved accuracy over the radiation condition.

Relaxation methods have been found to be one of the best methods in comparative numerical studies (e.g. Roed and Cooper (1987); Palma and Matano (1998); Nycander and Doos (2003)). However, the meaning of the relaxed solution in the sponge layer is questionable as it is not computed by any of the system's governing equations. In addition, parent model inaccuracies, if present, are introduced into the child grid solution not only on the nested boundary but throughout the extent of the sponge layer. During testing it was found that the sponge layer solution resulted in inaccuracies in the conservation and propagation of mass and momentum. The relaxation condition therefore produced a less accurate solution than the Dirichlet condition.

The boundary condition experimentations proved that the most stable and accurate model solution was achieved by the Dirichlet boundary condition; for this reason, it was implemented in the present model. Data was obtained from the parent solution, interpolated to the child grid and specified at the boundary for water elevations $\zeta$ and depth integrated velocity components in the $\mathrm{x}$ - and $\mathrm{y}$-directions, $U$ and $V$. Using these data, boundary values were also calculated for the water depths $H_{x}$ and $H_{y}$ and the volumetric flux components $q_{x}$ and $q_{y}$. The specification of elevations alone was sufficient to ensure conservation of mass and momentum when nested boundaries were located in flows primarily driven by mass. However, for more complex boundary flows governed more by momentum the specification of the additional variables was required to ensure conservation of both mass and momentum (see Nash (2010)).

\subsection{Boundary Formulation}

Upon initial implementation of the Dirichlet condition it was found that while mass conservation between the parent and child grids was relatively good, momentum conservation was poor. Investigation of this problem led to the more detailed analysis of the boundary formulation of the momentum equation. The formulation of the governing equations of flow is necessarily different for open boundary cells of a model than for interior grid cells. For example, the advective acceleration term in the x-direction momentum equation may be written in partial differential form as:

$$
\beta\left[\frac{\partial U q_{x}}{\partial x}+\frac{\partial U q_{y}}{\partial y}\right]
$$


where $\beta$ is the momentum correction factor for a non-uniform vertical velocity profile (i.e. the ratio of the true total momentum flux through a cross-sectional area and the momentum flux estimated from the depth-averaged flow velocity).

The calculation of the partial derivative $\left(\partial U q_{x} / \partial x\right)$ at grid cell $(x, y)$ using the central difference method on the model's space-staggered grid system requires values of $U$ and $q_{x}$ at the cell $(x, y)$ and at the two adjacent grid cells $(x-\Delta x, y)$ and $(x+\Delta x, y)$. For an interior grid cell this is not a problem, however, at a grid cell on the boundary of the model domain the adjacent external grid cell does not exist. At open boundary grid cells, such normal derivatives are therefore typically set to zero; hence, the boundary cannot completely model the effect of the exterior environment on the internal solution.

Regarding a nested domain, the situation is somewhat different; the exterior environment is known. The boundary formulations were thus modified so that the normal derivatives were computed as for an internal grid cell. The missing external grid cells were included in the domain as ghost cells and were incorporated in the modified boundary formulations. Using this technique, nested boundaries, in effect, become part of the interior domain or in other words, internal boundaries. To demonstrate, the finite difference formulation of the derivative $\left(\partial U q_{x} / \partial x\right)$ previously set to zero at an open boundary cell was instead calculated, using the ghost cells, as:

$$
\begin{aligned}
\frac{\partial U q_{x}}{\partial x}= & {\left[\frac{[U(x+\Delta x, y)+U(x, y)]}{2} \cdot \frac{\left[q_{x}(x+\Delta x, y)+q_{x}(x, y)\right]}{2}\right.} \\
& \left.-\frac{[U(x, y)+U(x-\Delta x, y)]}{2} \cdot \frac{\left[q_{x}(x, y)+q_{x}(x-\Delta x, y)\right]}{2}\right]
\end{aligned}
$$

The CG boundary configuration developed is shown in Figure 2. In the diagram, water elevations are indicated by $\bullet$ while velocities and volumetric fluxes are indicated by $\rightarrow$. Large symbols relate to the parent grid and small symbols to the child. It can be seen that the boundary configuration consists of two rows/columns of CG cells; the internal boundary cells and the adjacent exterior ghost cells. For clarity, child grid variables are only shown for these cells. Boundary data, interpolated from the PG, are specified at both the ghost cells and the internal boundary cells. Ghost cell data are only required for the computation of the derivatives of the components of velocity and volumetric flux normal to the boundary, thus only normal velocity and flux components are specified. For the internal boundary cells, all variable values are specified. Taking the example of the boundary parallel to the J-axis in Figure 2, normal velocities and fluxes, $U$ and $q_{x}$ respectively, are specified along interface ' $\mathrm{a}$ ' for the ghost cells and interface 'c' for the internal boundary cells. Water surface elevations, $\zeta$, and tangential velocity and flux components, $V$ and $q_{y}$ respectively, are also specified along interface ' $\mathrm{b}$ ' for the internal boundary cells. 


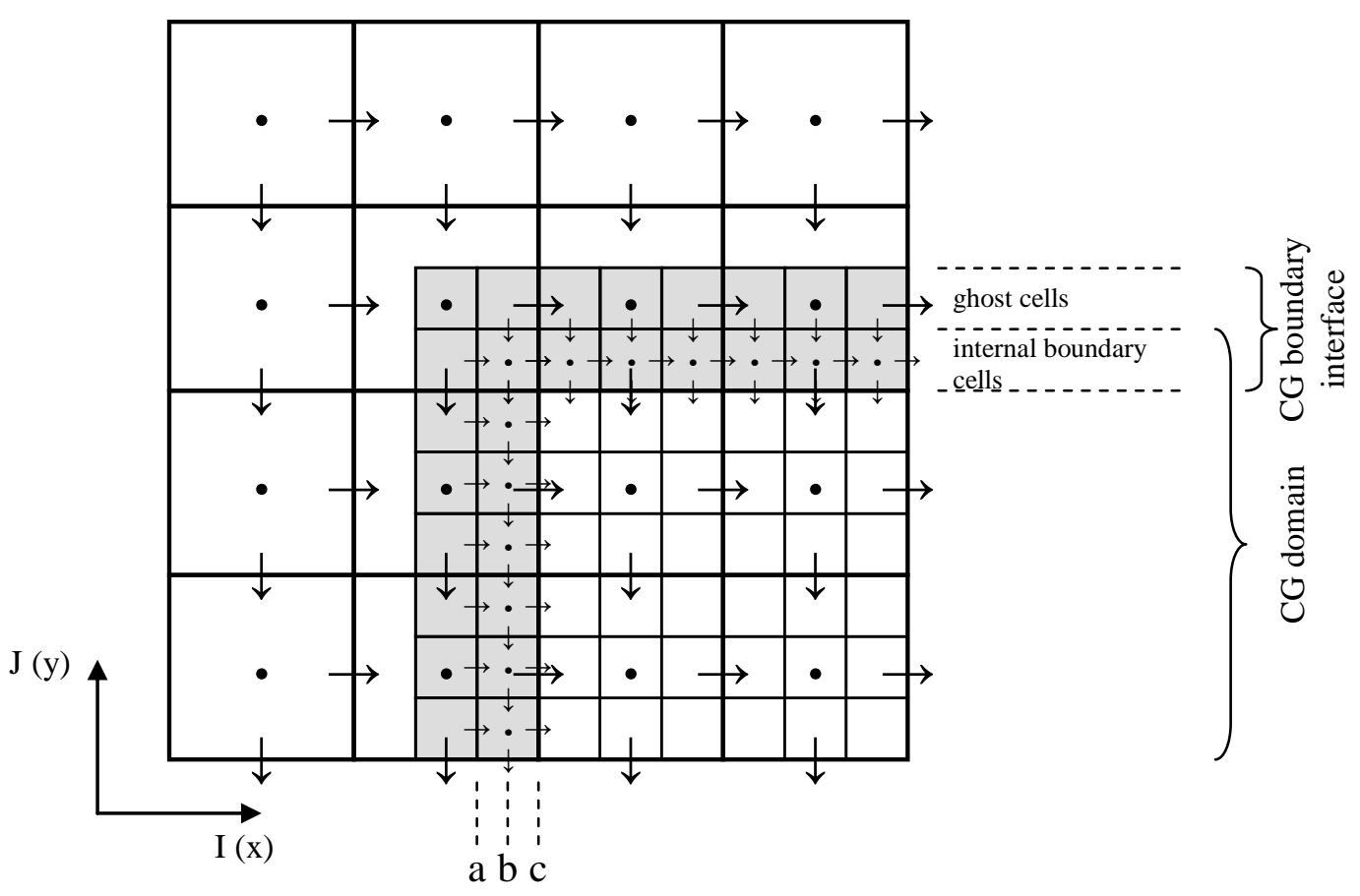

Fig 2 Grid configuration for a 3:1 nesting ratio showing the CG boundary interface (shaded).

Fictitious grid cells (see Fox and Maskell (1995) and Blayo and Debreu (2008)) similar to ghost cells have previously been used in nesting algorithms. The main difference between these approaches and that presented herein is the configuration of the CG boundary interface; this difference is demonstrated graphically in Figure 3. Based on interpretation of the literature, it is the Authors' understanding that all of the previous ghost cell approaches employ the CG boundary configuration shown in Figure $3 \mathrm{a}$ where PG boundary data is only specified to a single column/row of cells outside of the CG domain - the ghost cells. Data values at all grid cells within the CG domain are computed by the CG model. The calculation of normal derivatives at the CG boundary is therefore equally influenced by the PG boundary data and the computed CG solution. In the Authors' internal boundary approach, PG boundary data is specified to both the ghost cells outside the CG domain and to the internal boundary cells. Although the internal boundary cells are included in the CG domain the computation of their data values by the CG model is prevented; the PG forcing is therefore strictly imposed at these cells. The approach also ensures that the calculation of normal derivatives at the CG boundary is more strongly influenced by the parent grid data; this is consistent with the strict imposition of the PG forcing.

The implementation of the internal boundary in the nested model developed by the Authors was found to significantly improve the conservation of mass and, more importantly, momentum between the parent and child grids. Model results presented in Section 4.2 demonstrate the high conservation levels achieved by the Authors' consistent approach. 
(a)

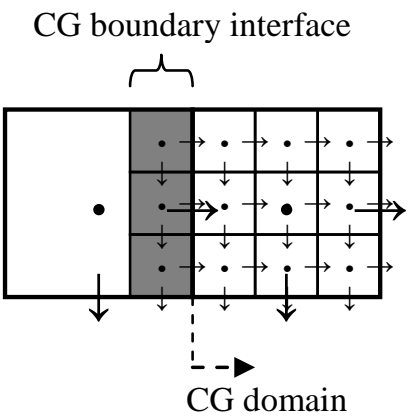

(b)

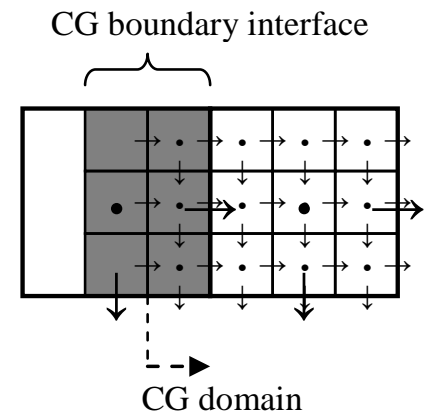

Fig 3 CG boundary configurations used in (a) previous nesting approaches incorporating ghost cells and (b) the Authors' ghost cell approach (shaded cells indicate PG data specification).

\subsection{Flooding and Drying on the Boundary}

The flooding and drying routine used in the model was originally developed and incorporated in DIVAST by Falconer and Chen (1991). The present research was not concerned with the performance of the flooding and drying routine itself - the routine has previously been extensively tested in both idealised channels and natural estuaries and has been shown to be both stable and accurate (Falconer and Chen, 1991). Rather, the research was concerned with the stability of a nested boundary located in an area of flooding and drying. The stability of a nested boundary in such an area will depend on the quality of the boundary data obtained from the parent grid and how those data are assimilated into the child grid.

The improvements in mass and momentum conservation, resulting from the internalisation of the nested boundary, were found to improve the performance of the moving boundary. However, significant errors were still found to occur near the boundary interface in those areas subject to flooding and drying. The reason for these errors was found to be a combination of the flooding and drying routine and the boundary interpolation scheme. In the model, when a grid cell dries out velocity components are set to zero and water level is maintained at the existing level immediately prior to drying. During interpolation of the child grid boundary data, parent grid cells which had dried out were being used in the interpolation procedure resulting in spurious boundary data values. An adaptive linear interpolation scheme was therefore designed and implemented in the model.

Figure 4 graphically illustrates how the adaptive linear interpolation scheme operates. Take the example of a parent grid cell $\left(i_{p}, j_{p}\right)$ located on a child grid boundary interface for which the enclosed child grid cell values are required. If one of the parent grid cells to either side of $\left(i_{p}, j_{p}\right)$ is dry, then the other adjacent parent grid cell is used in the linear interpolation formula (Figure $4 a$ and $4 b$ ); if both of the adjacent parent grid cells are dry then a zeroth-order interpolation scheme is applied (Figure 4c) and finally, if the enclosing parent grid cell $\left(i_{p}, j_{p}\right)$ is dry then all enclosed child grid cells are deemed dry and their variable values treated accordingly (Figure $4 \mathrm{~d}$ ). 


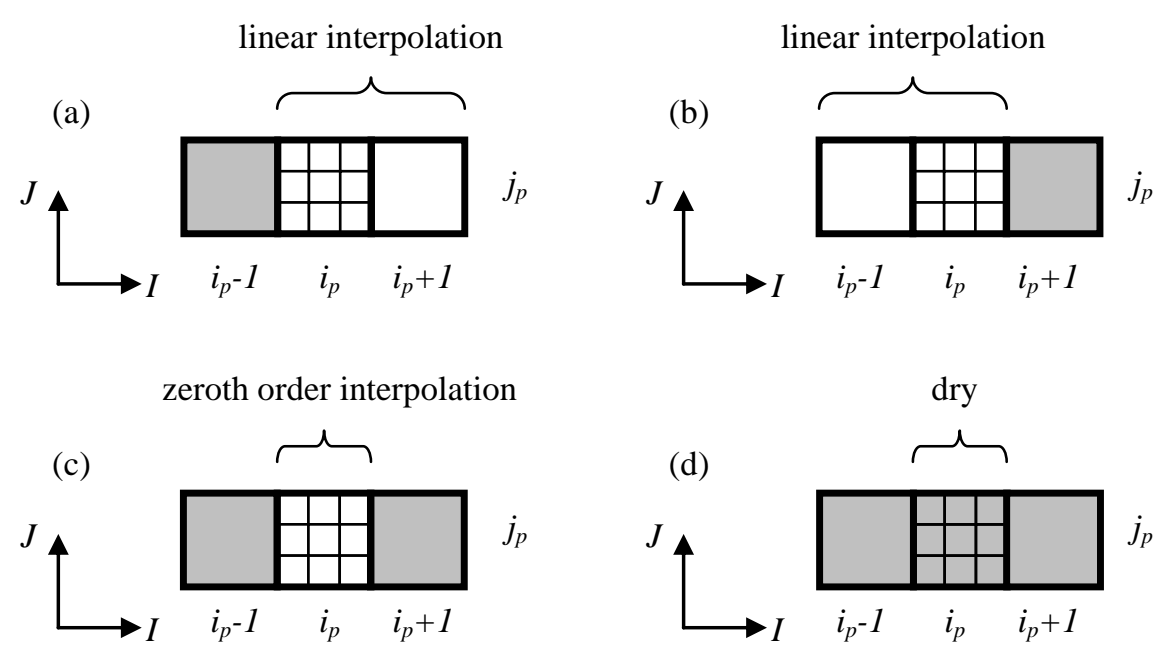

Fig 4 Graphical illustration of the adaptive linear interpolation scheme (shaded cells are dry)

\section{Boundary Results}

For each test case, two models were developed: a nested model and a single grid (SG) model. The nested model yielded two solutions for the AOI, the parent solution and the child solution. The SG model had the same extents as the PG model but was resolved at the same high resolution as the CG model; this allowed like-for-like comparisons with the $\mathrm{CG}$ in the AOI. The SG solution was termed the reference solution. Model performance was assessed by comparing PG and CG solutions with the reference solution. The determination of nested model accuracy in this way is a standard approach in cases where an analytic solution of the test case does not exist. For example, this method has been used by Spall and Holland (1991), Ginis et al. (1998), Blayo and Debreu (1999) and Rowley and Ginis (1999).

The performance of the nested boundary was primarily determined by analysis of net fluxes of mass and momentum across the boundary interface whilst the accuracy of the model solution at particular grid points was assessed by comparing timeseries of model variables. On the other hand, the accuracy of the domain-wide solution was determined using an original mathematical approach to error quantification the tidally-averaged relative error field, $R E_{T}$ (Nash, 2010). This approach allows the simultaneous analysis of spatial and temporal errors in a model solution in a single spatial distribution plot. The errors in both the PG and CG solutions are quantified by calculating $R E_{T}$ for both solutions relative to the reference solution. Spatial distributions of model variables are output at regular time intervals (snapshots) for each domain and, taking the example of the PG solution the tidally-averaged relative error at a grid cell $(i, j), R E_{T}(i, j)$, expressed as a percentage is then calculated as:

$$
R E_{T}(i, j)=\frac{\sum_{n=1}^{N}\left|\phi_{p}^{n}(i, j)-\phi_{s}^{n}(i, j)\right|}{\sum_{n=1}^{N} \phi_{p}^{n}(i, j)} .(100)
$$


where $\phi_{p}^{n}(i, j)$ and $\phi_{s}^{n}(i, j)$ are the hydrodynamic variables calculated by the PG and SG models respectively at grid cell $(i, j)$ and at the output time of snapshot $n . N$ is the total number of output times. To compute the tidally-averaged relative error field for an AOI equation (6) must be applied to each grid cell within the AOI. For a $12.5 \mathrm{hr}$ tidal cycle, tests established that $N=25$ was the lowest number of datasets required to give an accurate approximation of $R E_{T}$ at a particular grid point (see Nash (2010) for more details). Datasets were therefore output at half-hourly intervals over the course of a single tidal cycle.

\subsection{Boundary Operator}

The boundary operator was developed and tested using an idealised rectangular harbour with constant bed slope (see Figure 5). The harbour dimensions were $12 \times 6 \mathrm{~km}$ and the bed depth decreased from $10 \mathrm{~m}$ at the open boundary to $4 \mathrm{~m}$ at the back of the harbour. For simplicity a single nested grid was used at a 3:1 nesting ratio. The parent model used a grid spacing of $120 \mathrm{~m}$ and a timestep of $120 \mathrm{~s}$ while the child model used a grid spacing of 40m and a timestep of 40s. The CG boundary (A-A in Figure 5) spanned the full width of the harbour and was located exactly halfway along the longitudinal axis; the CG domain therefore comprised the inner half of the harbour. A tide of constant period (12.5hrs) and range (3m) was used as the model forcing.

A harbour wall was included in the model to induce momentum driven flows. The CG boundary was intentionally located in an area where flows were significantly influenced by momentum. Unfortunately, this also corresponded to a location of high parent model inaccuracy meaning that BS errors were introduced to the child model; however, the choice of location was judicious in developing a rigorous test of the boundary operator.
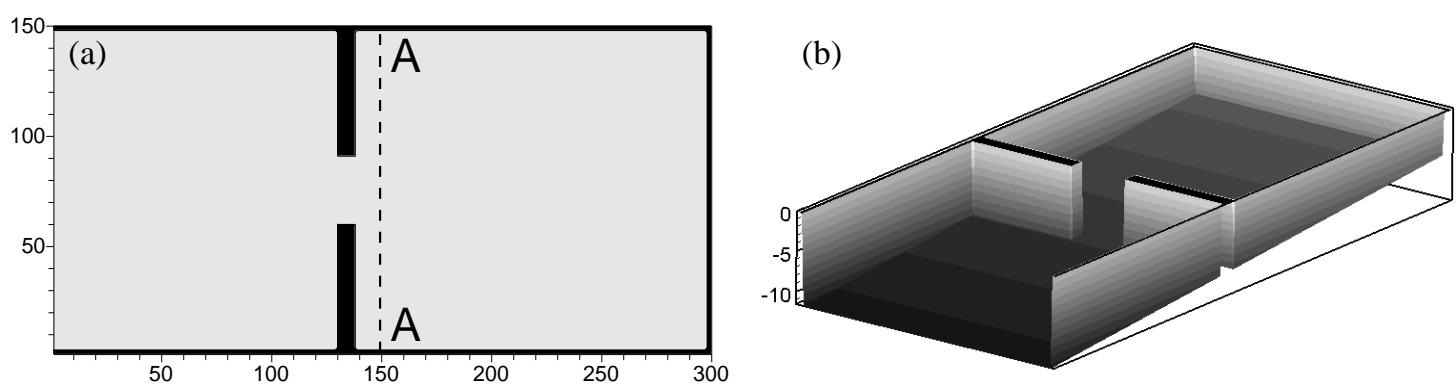

Fig 5 (a) Plan view and (b) 3d view of model domain (depths in metres)

\section{Spatial Interpolation}

Conservation of mass and momentum between parent and child grids is directly affected by the accuracy of the spatial interpolation scheme. Performance of four different schemes was assessed by analysing net fluxes of mass and momentum across the nested boundary interface (i.e. in the direction normal to the boundary interface). Child model fluxes were computed and compared with the corresponding fluxes from the parent model. Comparisons for a full tidal cycle are shown in Figures 6 and 7; the errors in child model flux relative to parent flux are also shown as these errors are not easily apparent from the flux 
graphs due to the scales involved. Fluxes were output immediately after the spatial interpolation of the boundary data so that any error could only be attributed to the interpolation scheme.
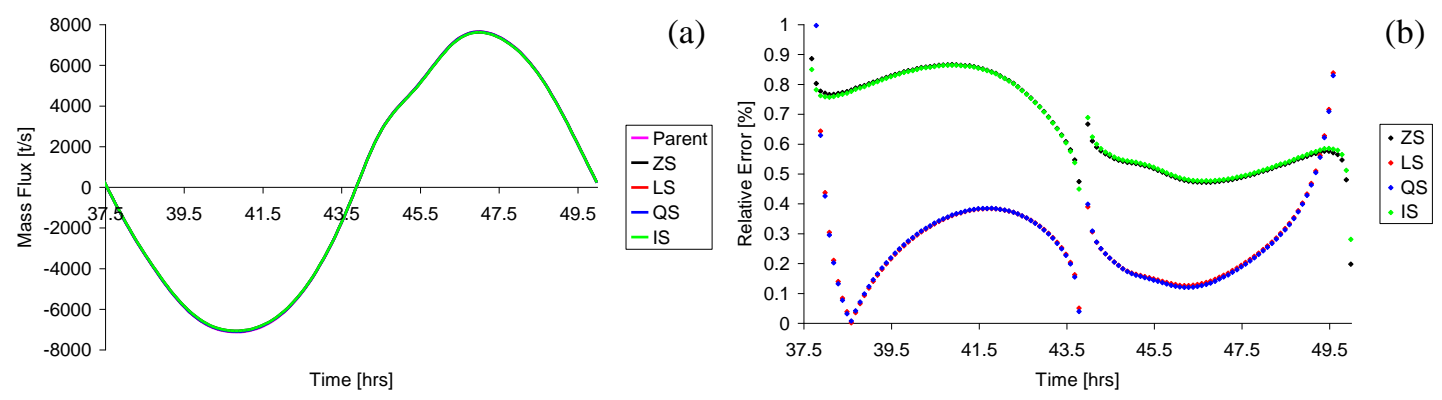

Fig 6 (a) Boundary mass fluxes and (b) errors relative to parent model
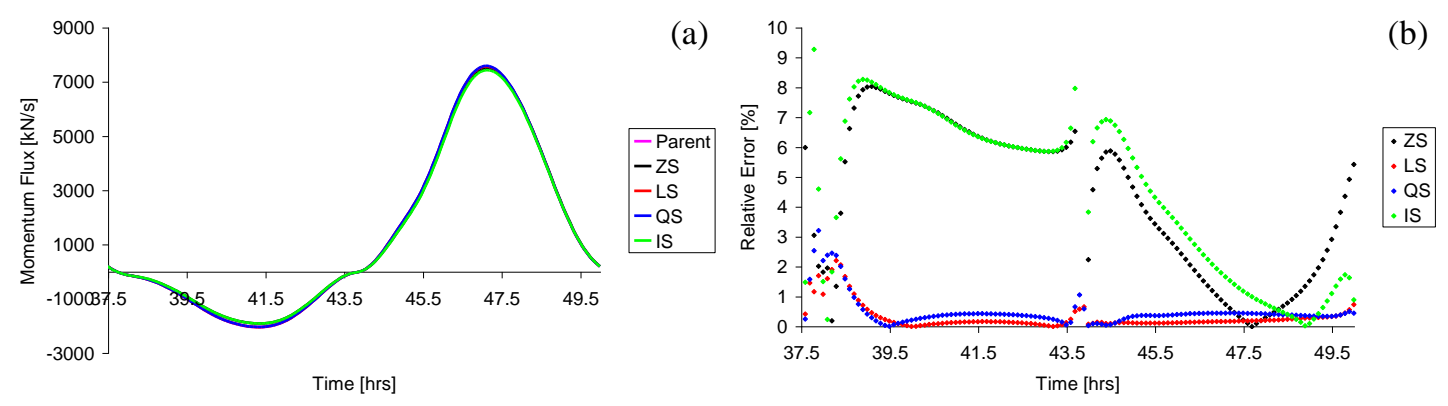

Fig 7 (a) Boundary momentum fluxes and (b) errors relative to parent model

The LS and QS were found to achieve similarly high levels of conservation. The ZS and IS also achieved similar, but lower, levels of conservation. The superior performance of the LS and QS was attributed to their mass-conservation properties. In the case of the LS conservation was naturally inherent to the scheme but in the case of the QS conservation was ensured by the introduction of a mass conservation parameter following Clark and Farley (1984).

Overall, the LS achieved the highest levels of conservation of both mass and momentum; the tidallyaveraged errors were acceptably low at $0.27 \%$ for mass and $0.32 \%$ for momentum. While the QS produced almost the same level of mass conservation, the level of momentum conservation was slightly lower than that of the LS. The results from the analysis agree with literature; Alapaty et al. (1998) state that higher-order schemes often lead to spurious oscillations of the interpolated variable in regions of sharp gradients and are thus less accurate than linear schemes. It should be noted that the highest errors were observed at the times of near zero fluxes and were therefore not significant in terms of conservation.

\section{Boundary Formulations}

The effect of the internal boundary formulation on model performance was also best demonstrated by the comparison of fluxes across the boundary interface. Figures 8 and 9 compare the boundary fluxes of mass 
and momentum from the child model, before and after the incorporation of the ghost cells, with those from the parent model. The time of output of the fluxes differed to those shown in Figures 6 and 7; in this case fluxes were output at each timestep following the solution of the governing equations of flow.

The ghost cell approach to boundary formulation significantly improved momentum conservation between the parent and child grids. The tidally-averaged error in boundary momentum flux decreased from $10.5 \%$ prior to the implementation of the internal boundary to $0.4 \%$ afterwards. The error in mass conservation, although already quite low prior to implementation, also decreased from $0.6 \%$ to $0.3 \%$. The improvement in mass and momentum conservation led to significant improvements in the accuracy of the nested model.
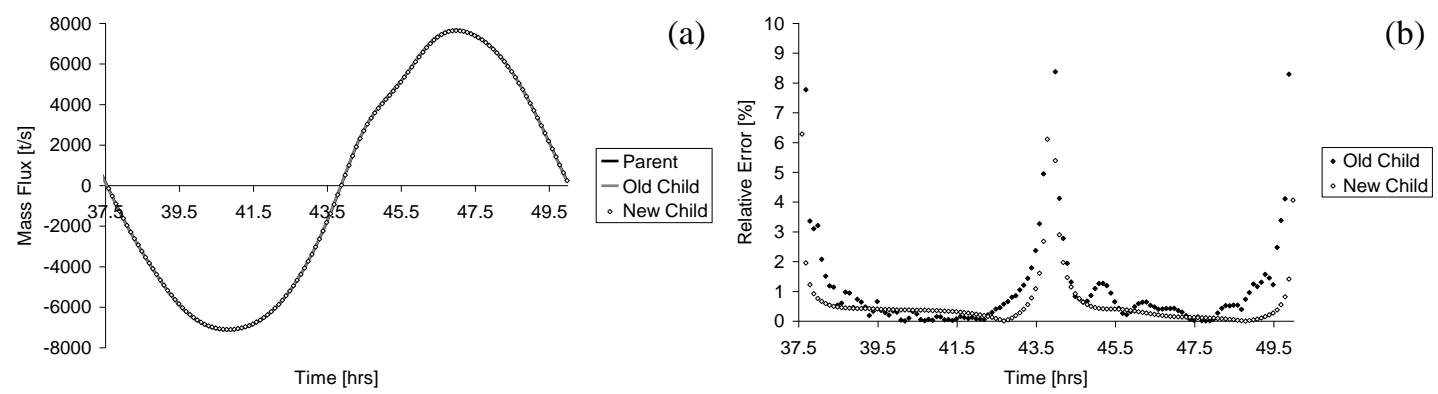

Fig 8 (a) Boundary mass fluxes and (b) errors relative to parent model
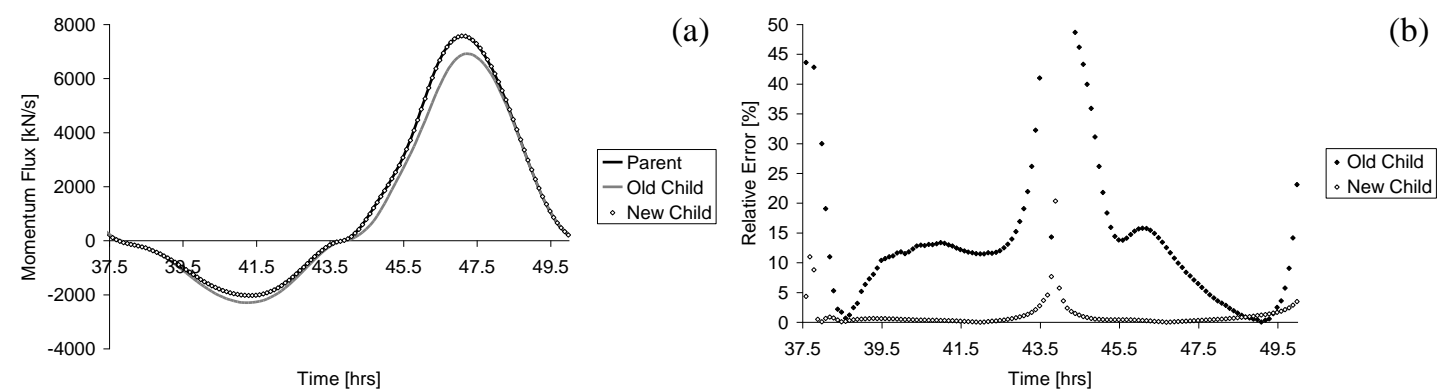

Fig 9 (a) Boundary momentum fluxes and (b) errors relative to parent model

\subsection{Moving Nested Boundary}

The new approach to the moving boundary problem was tested on an idealised harbour which was subject to flooding and drying (see Figure 10). The parent and child grid extents were the same as those used in the rectangular harbour experiment. The models were forced with a tide of constant period (12.5hrs) and range $(4 \mathrm{~m})$. In order to properly test the moving boundary, the harbour bed was shaped to ensure that flooding and drying occurred both inside the CG domain and on the CG boundary (A-A in Figure 10a). Figure 11 shows a bathymetric cross-section along the CG boundary A-A. From the diagram, it can be seen that approximately one third of the boundary's length is dry at low tide. 

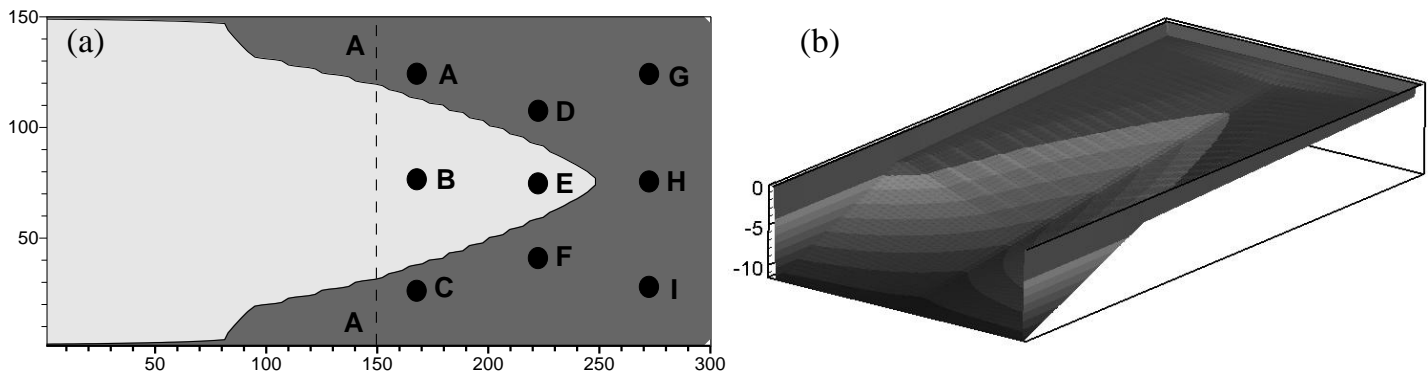

Fig 10 (a) Plan view of model domain showing CG boundary A-A and timeseries locations (A-I), and (b) $3 \mathrm{~d}$ view of model domain (depths in metres)

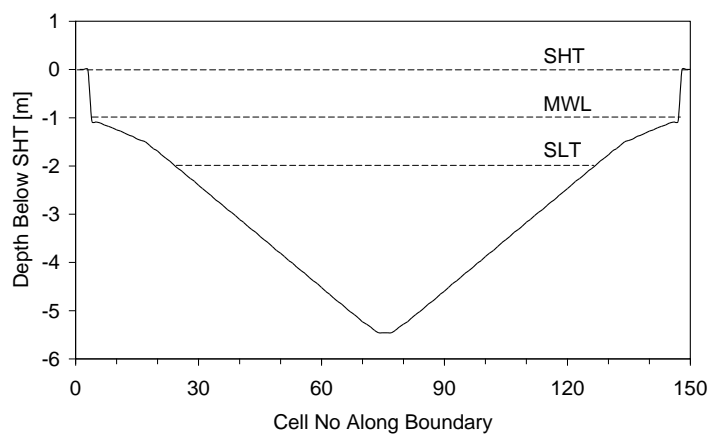

Fig 11 Bathymetric cross-section along CG boundary (SHT = spring high tide; MWL = mean water level; SLT $=$ spring low tide).

The improvement in the simulation of the moving nested boundary, resulting from the implementation of the internal dynamic boundary, was best demonstrated by examination of the $R E_{T}$ in $C G$ water elevations and current velocities. Figure 12 compares the $R E_{T}$ in $C G$ water elevations and current velocities computed by the nested model using the standard open boundary configuration with those computed by the nested model using the dynamic internal boundary. To demonstrate the improvement in accuracy achieved by the $\mathrm{CG}$ model, the $R E_{T}$ in the $\mathrm{PG}$ solution is also shown. It can be seen that whilst using the standard open boundary configuration resulted in an improvement in accuracy in the nested domain, large errors still occurred on and near the boundary in those areas subject to flooding and drying. This was due to the poor conservation of mass and momentum and the use of data values from dry PG cells in the interpolation of the CG boundary data. Errors can also be seen just inside the central section of the boundary; these were also a result of the poor conservation of momentum resulting from the simplified standard open boundary formulations. Neither type of error occurred when the dynamic internal boundary was used; as a result, the accuracy of the CG solution was similar to that of the SG reference solution with $R E_{T}$ in both elevations and velocities very close to zero across the full CG domain. It should be noted that the elevated relative errors at the back of the harbour in Figure $12 \mathrm{f}$ represented absolute errors of less than $0.001 \mathrm{~m} / \mathrm{s}$; these relative errors were therefore insignificant. 

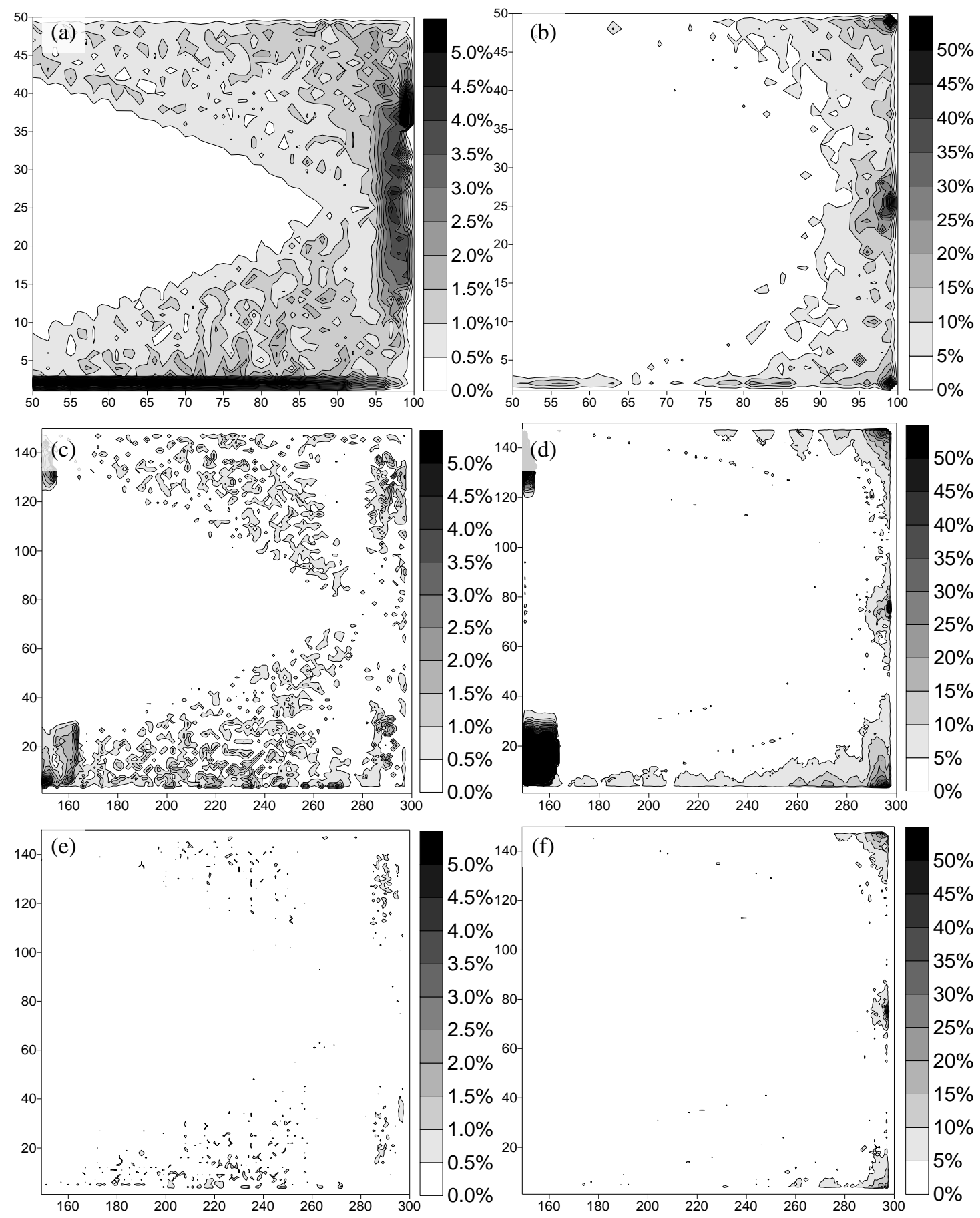

Fig 12 (a, b) $R E_{T}$ in PG elevations and velocities respectively, (c, d) $R E_{T}$ in CG elevations and velocities, respectively, for standard CG boundary configuration and (e, f) $R E_{T}$ in $C G$ elevations and velocities, respectively, for dynamic internal CG boundary configuration.

\section{Application to a Natural Harbour}

The most important elements of nested model development were the application of the model to a natural coastal system and the assessment of its performance. Cork Harbour, located on the southwest coast of Ireland, was chosen as the test case for model application. The Harbour, shown in Figure 13a, is one of 
the largest sea inlets in Ireland, with just under 120 miles of coastline. It is a macro-tidal estuary with typical spring tide ranges of $4.2 \mathrm{~m}$. The deepest point is $29 \mathrm{~m}$ below mean water level (MWL) and occurs in the entrance channel to the Lower Harbour while the average water depth is $8.4 \mathrm{~m}$ below MWL. At low water, extensive areas of mud- and sand-flats are exposed throughout the Harbour; it was primarily for this reason that it was chosen as a test case. In addition, the complex coastline and bathymetry of the region mean that the accuracy of a numerical model is highly dependent on the spatial resolution.

The domain chosen for nesting was the Owenboy Estuary; the extents of the domain and the AOI are shown in Figure 13b. The region was chosen as it has an extensive inter-tidal zone with large areas of the wetted domain being exposed at low spring tide. Most importantly, flooding and drying was prevalent on both open boundaries $\mathrm{OB}_{\mathrm{X}}$ and $\mathrm{OB}_{\mathrm{Y}}$. Indeed, almost half of the boundary $\mathrm{OB}_{\mathrm{Y}}$ dries out at low tide; this can be seen in Figure 14 which shows bathymetric cross-sections of both open boundaries.

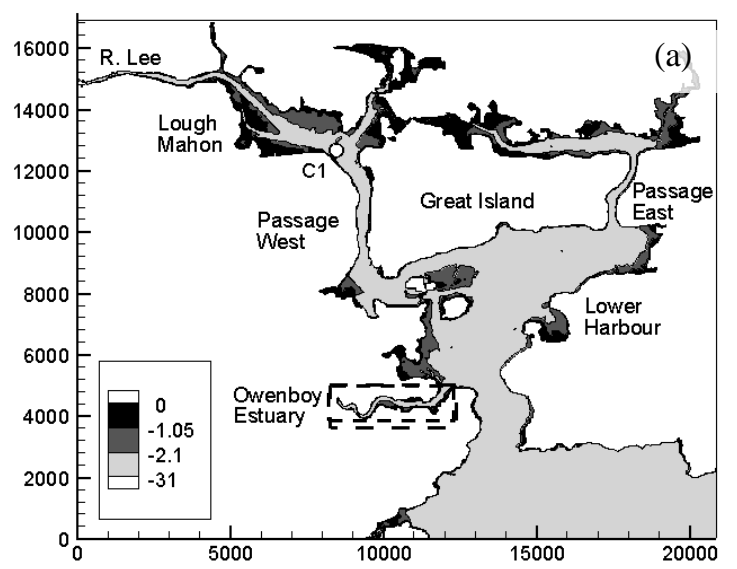

Fig 13 (a) Cork Harbour domain and AOI (dashed) and (b) extents of nested domain with AOI (dotted). Mudflats on a spring tide are indicated by the $-2.1 \mathrm{~m}$ contour
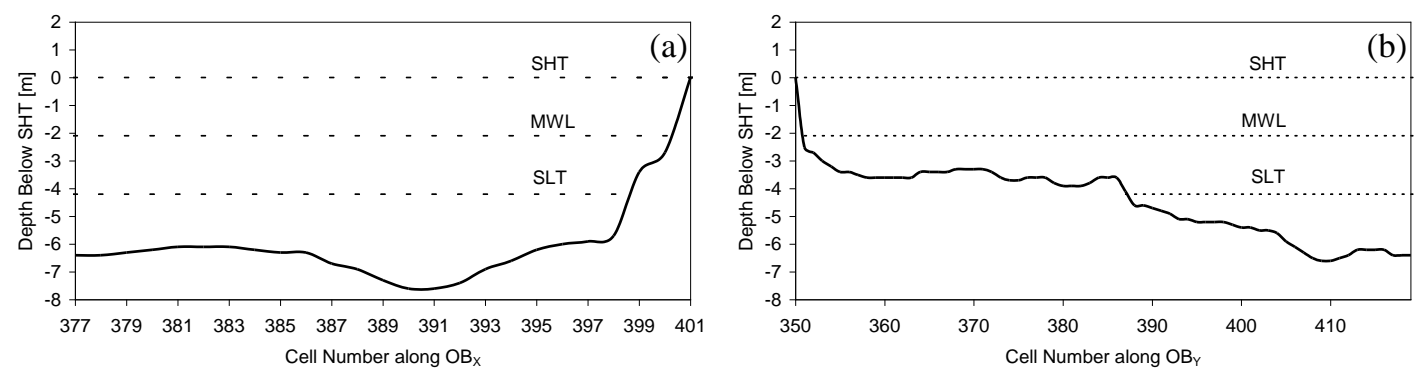

Fig 14 Bathymetric cross-sections along (a) boundary $\mathrm{OB}_{\mathrm{X}}$ and (b) boundary $\mathrm{OB}_{\mathrm{Y}}(\mathrm{SHT}=$ spring high tide, $\mathrm{MWL}=$ mean water level, $\mathrm{SLT}=$ spring low tide)

The parent model comprising the full domain of Figure 13a was resolved at a grid spacing of $90 \mathrm{~m}$ and a timestep of 18s. A 3:1 nesting ratio was used, thus the child model was resolved at a grid spacing of $30 \mathrm{~m}$ and a timestep of $6 \mathrm{~s}$. The accuracy of the PG and CG solutions was determined relative to a reference 
solution - the solution from a SG model with the same domain extents as the parent grid and the same high resolution as the child grid. The models were run for a constant spring tide so that the areas of flooding and drying were maximised. The SG model had been extensively calibrated and validated during previous research (see Costello et. al. (2001)). To demonstrate that the high resolution did indeed give improved model accuracy, Figure 15 compares current velocities computed by the high resolution SG model and the low resolution PG model with measured velocities at point C1 (see Figure 13a) over a single spring tidal cycle. It can be seen that the PG model erroneously computed a period of drying at low water (indicated by the zero velocities); this was not reflected in the measured data, nor was it computed by the SG model. The improved accuracy of the SG solution is clear to be seen.

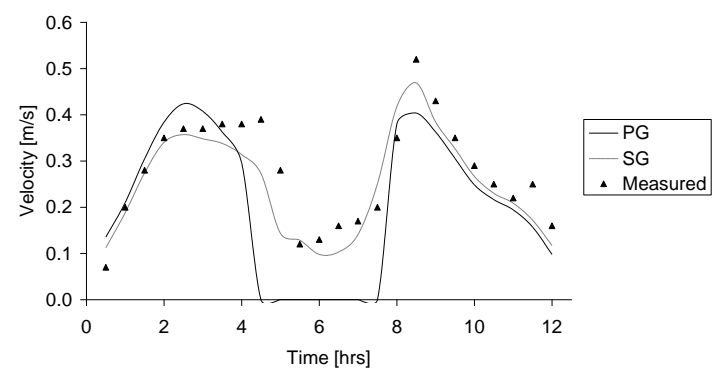

Fig 15 Comparison of computed and measured velocities at point $\mathrm{C} 1$ in Figure 13a.

\subsection{Model Accuracy}

In order to assess model performance, analysis of model results was only carried out for the AOI. Figure 16 compares the $R E_{T}$ in $\mathrm{PG}$ and CG water elevations in the AOI. Typically, water elevations will tend to be quite accurate irrespective of model resolution. Overall, errors were quite low with the areas of largest errors occurring in the inter-tidal areas (see Figure 13b). The nested model achieved a significant reduction in model error. While the $R E_{T}$ in the parent model exceeded $1 \%$ in $47 \%$ of the AOI, this level was only exceeded in the child model in $4 \%$ of the AOI. The average $R E_{T}$ value for the AOI was found to decrease from $8.3 \%$ in the parent model to $0.6 \%$ in the child.

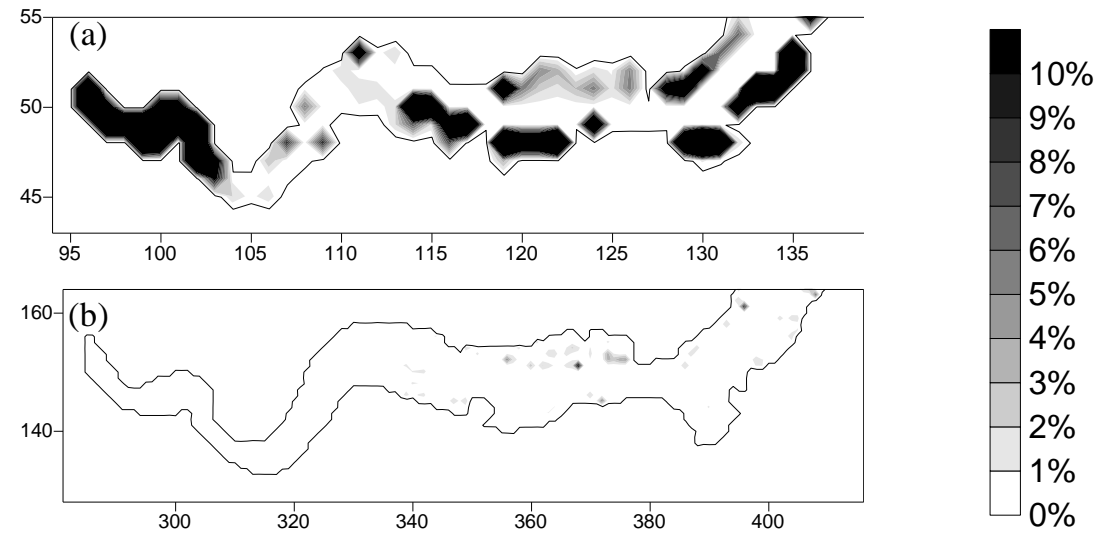

Fig $16 R E_{T}$ in water elevation in the AOI for (a) parent model and (b) child model 
The nested model also significantly improved the accuracy of the current velocity computations. Figure 17 compares the $R E_{T}$ in PG and CG velocities in the AOI. Firstly, it can be seen that errors in the parent model were much greater for current velocities than for water surface elevations; this was as expected. Secondly, the $R E_{T}$ in the parent model velocities exceeded $5 \%$ in $86 \%$ of the AOI; by contrast this level was never exceeded in the child model. In general, the average $R E_{T}$ in velocities for the AOI decreased from $45 \%$ in the parent model to $0.1 \%$ in the child.

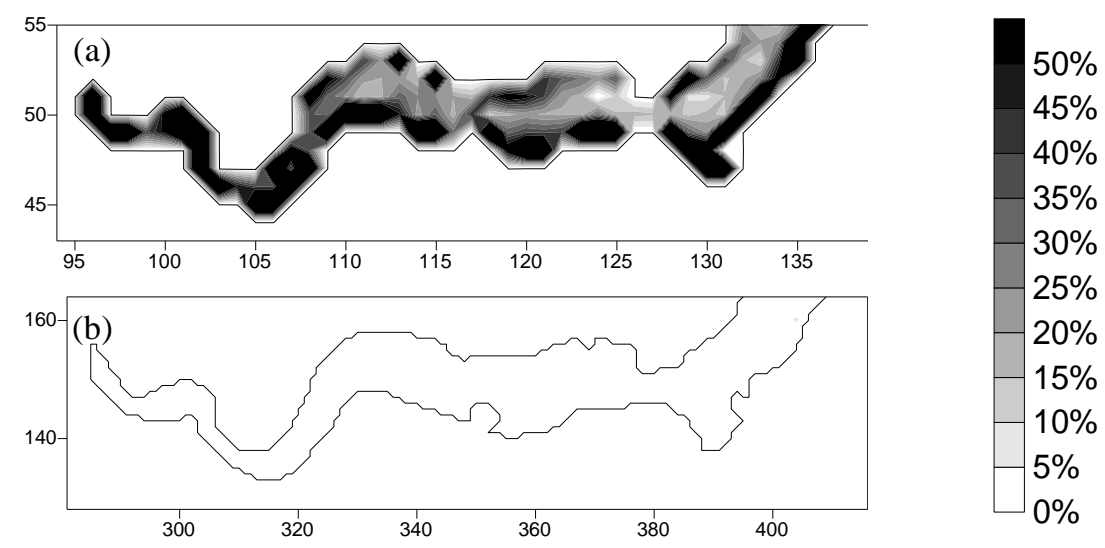

Fig $17 R E_{T}$ in current velocity in the AOI for (a) parent model and (b) child model

In order to verify the accuracy of the nested model, time series of water elevations and current velocity magnitudes were analysed at a number of different locations (see Figure 18). Due to space limitations, time series comparisons are only presented herein for points A (Figure 19) and C (Figure 20). Water elevations computed by the parent model were quite accurate for the most part, however, large inaccuracies were present in the current velocities; this agreed with the spatial error distributions. An improvement in the accuracy of water elevations in those areas subject to flooding and drying can be seen at location A. The lower resolution of the parent model was unable to resolve the drying of grid cells to the same level of accuracy as the high resolution model; a $0.5 \mathrm{~m}$ error was recorded in the level at which the grid cell was deemed to have dried out. In contrast, the child model was capable of resolving the intertidal region to the same accuracy as the single grid model.

Significant improvements in the accuracy of velocity computations were achieved by the child model. At point A the tidally-averaged error in velocities was reduced from $329 \%$ in the parent model to $3 \%$ in the child model; at point $\mathrm{C}$ the error was reduced from $25 \%$ to $2 \%$. The time series show that the nested model achieves a similar level of accuracy to the high resolution model. This was verified by using linear regression of the time series data (see Figure 21) to calculate the degree of correlation, $r^{2}$, between the CG and SG velocities. As can be seen, $r^{2}$ values of 0.99 and 1 were computed for the CG velocities at points $\mathrm{A}$ and $\mathrm{C}$ respectively. In addition to the high $r^{2}$ values, it can be seen from the equations of the CG trendlines that the slopes were equal to 1 and the intercepts were zero; this further demonstrates the high level of accuracy of the nested model. 


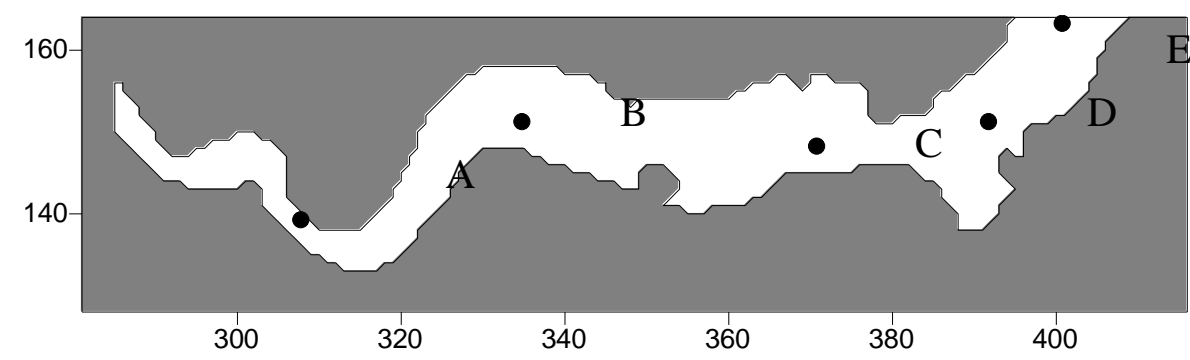

Fig 18 Time series locations in the AOI
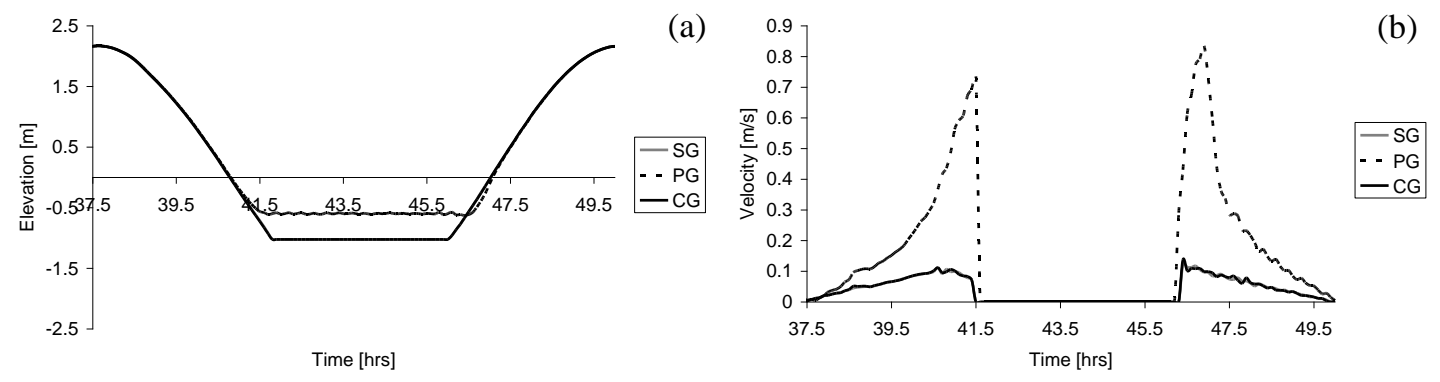

Fig 19 (a) Water elevations and (b) current velocities at point A (SG and CG coincident)
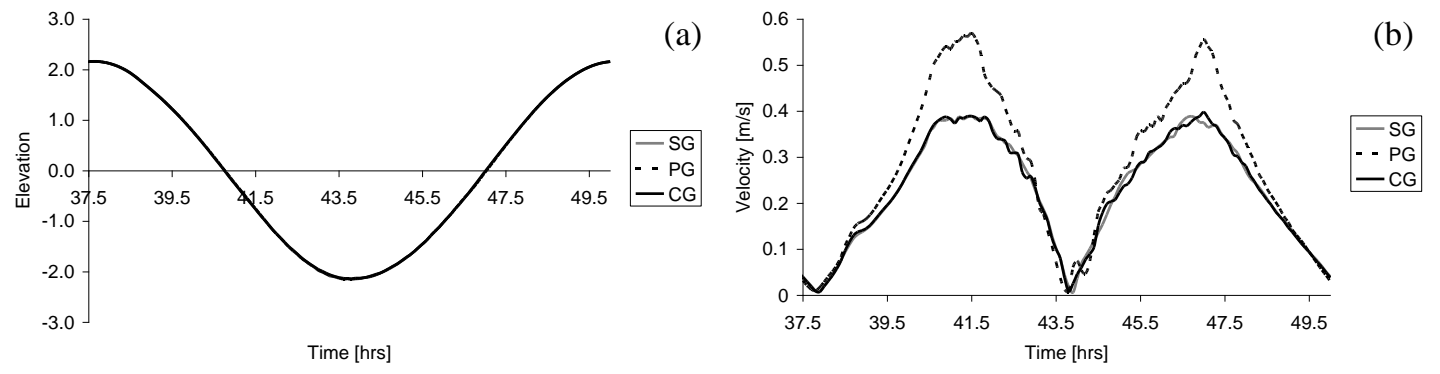

Fig 20 (a) Water elevations and (b) current velocities at point C (SG and CG coincident)
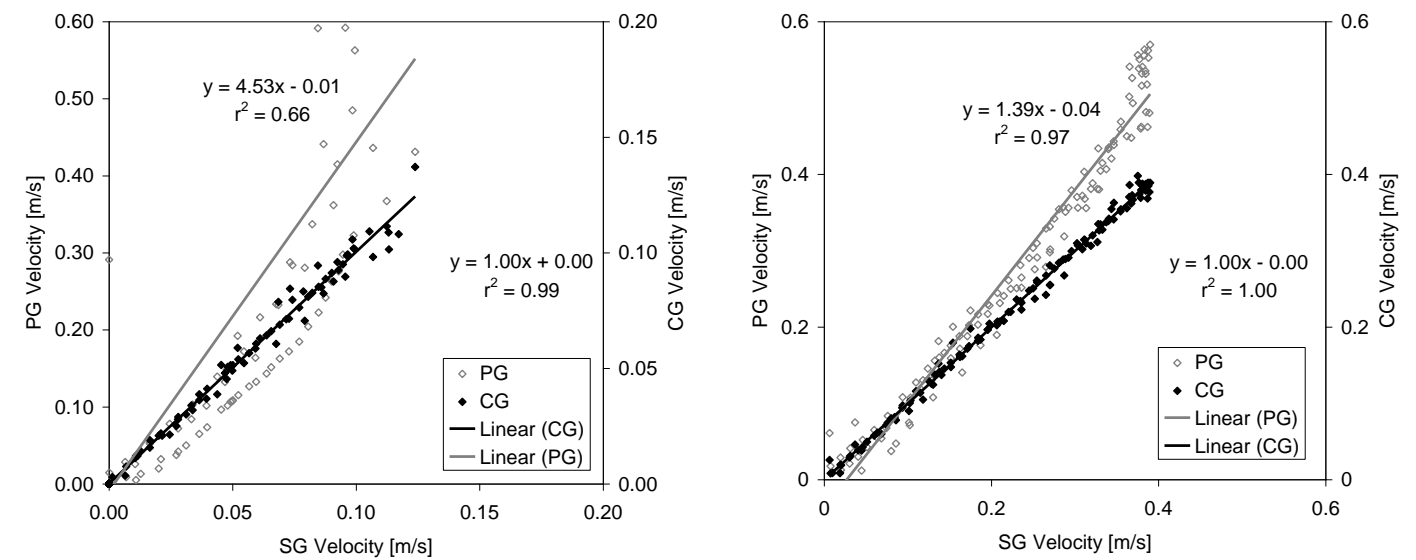

Fig 21 Linear regression analyses of velocity time series data showing trend-lines and $r^{2}$ values for (a) Point A and (b) Point C 


\section{Conclusions}

The Authors have developed a nested circulation model incorporating flooding and drying which has significant and practical applications in the modelling of coastal areas and, in particular, inter-tidal zones. The accurate modelling of inter-tidal zones requires a high resolution which can be cost prohibitive. The nested model allows high resolution modelling in an AOI but at a significantly lower computational cost than a high resolution single-grid model. For example, the application of the model to the Owenboy estuary presented herein resulted in a $92 \%$ saving in computation time over an equivalent large domain high resolution model. The model results demonstrated that the model not only offered improved accuracy over the lower resolution parent model but that the level of accuracy achieved in the AOI was similar to that of the high resolution model.

The nested modelling of inter-tidal zones requires the incorporation of moving nested boundaries to accommodate flooding and dying of boundary grid cells. Faced with this problem, nested models have traditionally either prohibited the flooding and drying of nested boundaries or omitted the process completely. The model developed by the Authors solves the moving boundary problem through the incorporation of ghost cells in the mathematical formulations of the nested boundaries. Nested boundary cells are formulated in a similar manner to interior grid cells by specifying ghost cells adjacent to the boundary and obtaining data for both the ghost cells and the internal boundary cells from the parent model. In combination with an adaptive linear interpolation scheme designed specifically for the model, nested boundaries are, in effect, converted into dynamic internal boundaries; this enables the stable flooding and drying of boundary grid cells.

Finally, errors are introduced to a nested model at the boundary interface; particular attention was, therefore, focused on the development of the boundary operator. A linear interpolation technique and a Dirichlet boundary condition were found to give the highest levels of conservation of mass and momentum between parent and child grids. The internalisation of the boundary was also found to significantly improve conservation at the boundary by allowing the boundary to better model the effects of the external environment on the internal hydrodynamics.

\section{Appendix A: Spatial Interpolation Formulae}

\section{Zeroth-order Interpolation Scheme (ZS):}

If $\phi_{i}$ represents the value of a parent grid variable at cell $i$, then $\phi_{k}$ for all the child grid cells within its confines can be written as:

$$
\phi_{k}=\phi_{i} \quad k=1, \ldots, m
$$

where $m$ is the number of child grid cells within the parent grid cell, i.e. $m=r_{s}{ }^{2}, r_{s}$ being the spatial nesting ratio. 


\section{Linear Interpolation Scheme (LS):}

The variable $\phi$ in a child grid cell $k$ which lies within the parent grid cell $i$ may be calculated as:

$$
\phi_{k}=\phi_{i-l}+\omega\left(\phi_{i}-\phi_{i-1}\right)
$$

with $\omega$, the proportional interpolation coefficient, further expressed as:

$$
\omega=\frac{2 k+r_{s}-1}{2 r_{s}}, \quad k=1, \ldots, r_{s}
$$

\section{Quadratic Interpolation Scheme (QS):}

For a given nesting ratio $r_{s}$, the interpolation of the parent grid cell variable $\phi_{i}$ to any child grid cell $k$ of any horizontal row of enclosed child grid cells may be specified as (adapted from Alapaty et al. (1998)):

$$
\phi_{k}=E_{i-1}^{k} \phi_{i-1}+E_{i}^{k} \phi_{i}+E_{i+1}^{k} \phi_{i+1}
$$

with the function $E$ further expressed as:

$$
\begin{aligned}
& E_{i-1}^{k}=\frac{\lambda_{k}\left(\lambda_{k}-1\right)}{2}+\alpha \\
& E_{i}^{k}=\left(1-\lambda_{k}^{2}\right)-2 \alpha \\
& E_{i+1}^{k}=\frac{\lambda_{k}\left(\lambda_{k}+1\right)}{2}+\alpha
\end{aligned}
$$

where $\lambda$ represents a normalised local coordinate pointing in the same direction as the global coordinate and whose origin coincides with the centre of the parent grid cell $i$. The value of $\lambda$ for the child grid cell $k$ is defined as:

$$
\lambda_{k}=\frac{(2 k-1) \Delta x_{c}-\Delta x_{p}}{2 \Delta x_{p}}, \quad k=1, \ldots, r_{s}
$$

and

$$
\alpha=\frac{1}{24}\left[\left(\frac{\Delta x_{c}}{\Delta x_{p}}\right)^{2}-1\right]
$$

$\Delta x_{p}$ and $\Delta x_{c}$ are the parent and child grid spacings respectively. The parameter $\alpha$ is introduced to ensure mass conservation following Clark and Farley (1984). 


\section{Inverse Distance Weighted Interpolation Scheme (IS):}

The variable $\phi$ in a child grid cell $k$ which lies within an enclosing parent grid cell is calculated as:

$$
\phi_{k}=\sum_{i=1}^{n} w_{i} \phi_{i} / \sum_{i=1}^{n} w_{i} \quad k=1, \ldots, m
$$

where $n$ is the number of parent grid cells used in the interpolation and $w_{i}$ is the weighting function. For the nested model $n=9$, i.e. the enclosing grid cell and the eight adjacent grid cells were used. The weighting function is written as:

$$
w_{i}=\frac{1}{d_{(k, i)}{ }^{2}}
$$

where $d_{(k, i)}$ is the distance from the child grid point $k$ to the parent grid point $i$. The weighting function varies from a value of unity at the child grid point of interest to a value approaching zero as the distance from the grid point increases.

\section{References}

Alapaty, K., Mathur, R. and Odman, T., (1998). Intercomparison of spatial interpolation schemes for use in nested grid models, Monthly Weather Review (126), pp. 243-249

Barth, A., Alvera-Azca'rate, A., Rixen, M. and Beckers, J-M, (2005). Two-way nested model of mesoscale circulation features in the Ligurian Sea. Progress in Oceanography 66, pp. 171-189.

Blayo, E. and Debreu, L., (2005). Revisiting open boundary conditions from the point of view of characteristic variables, Ocean Modelling (9), pp. 231-252.

Blayo, E. and Debreu, L., (2008). Two-way embedding algorithms: a review, Ocean Dynamics (58), pp. 415-428.

Clark, T.L. and Farley, R.D., (1984). Severe downslope windstorm calculations in two and three dimensions using anelastic interface grid nesting: a possible mechanism for gustiness, Journal of Atmospheric Science, Vol. 41, pp. 329-350.

Costello, M.J., Hartnett, M., Mills, P., O’Mongain, E., Collier, L., Johnson, M., Nash, S., Leslie, R., Berry, A., Emblow, C., Collins, A., and McCrea, M., (2001). Nutrient dynamics of two estuaries in Ireland: Wexford and Cork Harbours, Irish Environmental Protection Agency.

DHI Software, (2007). Mike 21 flow model: hydrodynamic module user guide, DHI Water and Environment.

Falconer, R.A. and Chen, Y.P., (1991). An improved representation of flooding and drying and wind stress effects in a 2-D tidal numerical model, Proceedings of Institution of Civil Engineers, Part 2, Research and Theory, Vol. 91, pp. 659-678.

Fox, A.D. and Maskell, S.J., (1995). Two-way interactive nesting of primitive equation ocean models with topography, Journal of Physical Oceanography 25 pp. 2977-2996. 
Ginis, I., Richardson, R.A. and Rothstein, L.M., (1998). Design of a multiply nested primitive equation ocean model, Monthly Weather Review 126, pp. 1054-1079.

Holt, J., Harle, K., Proctor, R., Michel, S., Ashworth, M., Batstone, C., Allen, I., Holmes, R., Smyth, T., Haines, K., Bretherton, D. and Smith, G., (2009). Modelling the global coastal ocean, Philosophical Transactions of the Royal Society A (367), pp. 939-951.

Koch, S.E. and McQueen, J.T., (1987). A survey of nested grid techniques and their potential for use within the MASS weather prediction model, NASA Technical Memorandum 87808, National Aeronautics and Space Administration, USA.

Korres, G. and Lascaratos, A., (2003). A one-way nested eddy resolving model of the Aegean and Levantine Basins: implementation and climatological runs. Annales Geophysicae 21, pp. 205-220

Leitão, P., Coelho, H., Santosa, A. and Nevesa, R., (2005). Modelling the main features of the Algarve coastal circulation during July 2004: a downscaling approach, Journal of Atmospheric \& Ocean Science, 10:4, pp. 421-462.

Marchesiello, P., McWilliams, J. and Shchepetkin, A., (2001). Open boundary conditions for long-term integration of regional oceanic models, Ocean Modelling (3), pp. 1-20.

Miyakoda, K. and Rosati, A. (1977). One-way nested grid models: the interface conditions and the numerical accuracy. Monthly Weather Review, 105, pp. 1092-1107

Nittis, K., Perivoliotis, L., Korres, G., Tziavos, C. and Thanos, I., (2006). Operational monitoring and forecasting for marine environmental applications in the Aegean Sea, Environmental Modelling \& Software (21), pp. 243-257.

Nash, S. (2010). Development of an adaptive mesh inter-tidal circulation model, PhD Thesis, College of Engineering \& Informatics, National University of Ireland Galway.

Nycander, J. and Doos, K., (2003). Open boundary conditions for barotropic waves, Journal of Geophysical Research, 108 (C5), pp. 3168-3187.

Palma, E.D. and Matano, R.P., (1998). On the implementation of passive open boundary conditions for a general circulation model: the barotropic mode, Journal of Geophysical Research, 103:C1, pp. 1319-1341. Pullen, J. and Allen, J.S., (2001). Modeling studies of the coastal circulation off the northern coast of California: statistics and patterns of wintertime flow, Journal of Geophysical Research, 106:C11, pp. 26,959-26,984

Roed, L.P. and Cooper, C., (1987). A study of various open boundary conditions for wind-forced barotropic numerical ocean models, in Three-dimensional Models of Marine and Estuarine Dynamics, edited by Nihoul, J.C.J. and Jamart, B.N., pp. 305-335, Elsevier.

Spall, M.A. and Robinson, A.R. (1989). A new hybrid coordinate open ocean primitive equation model, Mathematics and Computers in Simulation, 31, 241-269.

Spall, M.A. and Holland, W.R., (1991). A nested primitive equation model for oceanic applications, Journal of Physical Oceanography, Vol. 21, pp. 205-220.

Staneva, J., Stanev, E.V., Wolff, J.O., Badewien, T.H., Reuter, R., Flemming, B., Bartholoma, A. and Boldinge, K., (2009). Hydroynamics and sediment dynamics in the German Bight: a focus on 
observations and numerical modelling in the East Frisian Wadden Sea, Continental Shelf Research (29), pp. 302-319.

Zhang, Da-Lin, Hai-Ru Chang, N.L. Seaman, T.T. Wamer, and J.M. Fritsch, (1986). A two-way interactive nesting procedure with variable terrain resolution, in: Koch, S.E., McQueen, J.T., (1987). 